\title{
ST-elevation myocardial infarction due to acute occlusion of the right coronary artery and con- comitant very late stent thrombosis in the left circumflex artery
}

\author{
Henryk Dreger ${ }^{1}$, Karl Stangl ${ }^{1}$, Martin Möckel $^{2}$ \\ 1. Department of Cardiology and Angiology, Campus Charité Mitte, Charité-Universitätsmedizin Berlin, Germany. 2. \\ Division of Emergency Medicine and Department of Cardiology, Campus Virchow-Klinikum and Campus Charité Mitte, \\ Charité-Universitätsmedizin Berlin, Germany.
}

Correspondence: Henryk Dreger. Address: Department of Cardiology and Angiology, Campus Charité Mitte, Charité-Universitätsmedizin Berlin, Germany. Email: henryk.dreger@charite.de

Received: September 11, 2014

DOI : $10.5430 /$ crim.v2n1p41
Accepted: October 13, $2014 \quad$ Online Published: October 20, 2014

URL: http://dx.doi.org/10.5430/crim.v2n1p41

\begin{abstract}
We report on a 72-year-old patient who presented with a STEMI due to acute occlusion of the right coronary artery and concomitant late stent thrombosis in the left circumflex artery. Notably, acute thrombus formation occurred in stents that had been implanted in the LCx nine years before the current event. We conclude that STEMI patients are in a hypercoagulant state and therefore prone to very late stent thrombosis which requires adequate awareness of the interventionist.
\end{abstract}

\section{Keywords}

Myocardial infarction, Stent thrombosis, Percutaneous coronary intervention, Acute coronary syndrome

\section{I ntroduction}

In the majority of cases, ST-elevation myocardial infarction (STEMI) is caused by a complete occlusion of a major coronary vessel. Accordingly, even in the presence of multi-vessel disease, angiographic identification of the culprit lesion is usually not difficult in STEMI patients. In some patients, however, angiography reveals more than one occluded vessel. In these cases, the electrocardiogram (ECG) as well as the characteristic angiographic appearance of the occluded vessels must be used to distinguish the culprit lesion from chronic total occlusions.

Stent thrombosis is a rare but potentially fatal complication after percutaneous coronary interventions. Fortunately, the prevalence of stent thrombosis is rare after formation of a neo-intima. However, especially in patients in a hypercoagulant state, e.g. after major surgery, late stent thrombosis (i.e. more than one year after stent implantation) may occur. Here, we present a case with acute occlusion of the right coronary artery (RCA) and concomitant very late stent thrombosis in the left circumflex artery (LCx). 


\section{Case presentation}

A 72-year-old man with a history of coronary artery disease was admitted to our emergency department. In August of 2003, the patient had undergone coronary catheterization for stable angina pectoris. Coronary artery disease was diagnosed and two stents were deployed in the LCx. In October of 2003, the patient was symptomatic again and underwent double bypass surgery (left internal mammary artery to left anterior descending artery and a saphenous jump graft to the first and second marginal branch of the LCx).

The patient now presented in our emergency department with an acute episode of severe chest discomfort that had started at rest about one hour before admittance. Apart from frequent premature ventricular contractions he was in a stable clinical condition. Suspected myocardial infarction was confirmed by laboratory testing and ECG which showed elevated troponin levels and ST elevations in the inferior leads (see Figure 1) respectively. The following angiography revealed an acute occlusion of the RCA as well as a concomitant acute occlusion of the proximal LCx due to thrombus formation in the stents implanted nine years ago (see Figure 2). TIMI III flow was rapidly restored in both RCA and LCx by stent implantation and balloon dilation, respectively (see Figure 3).

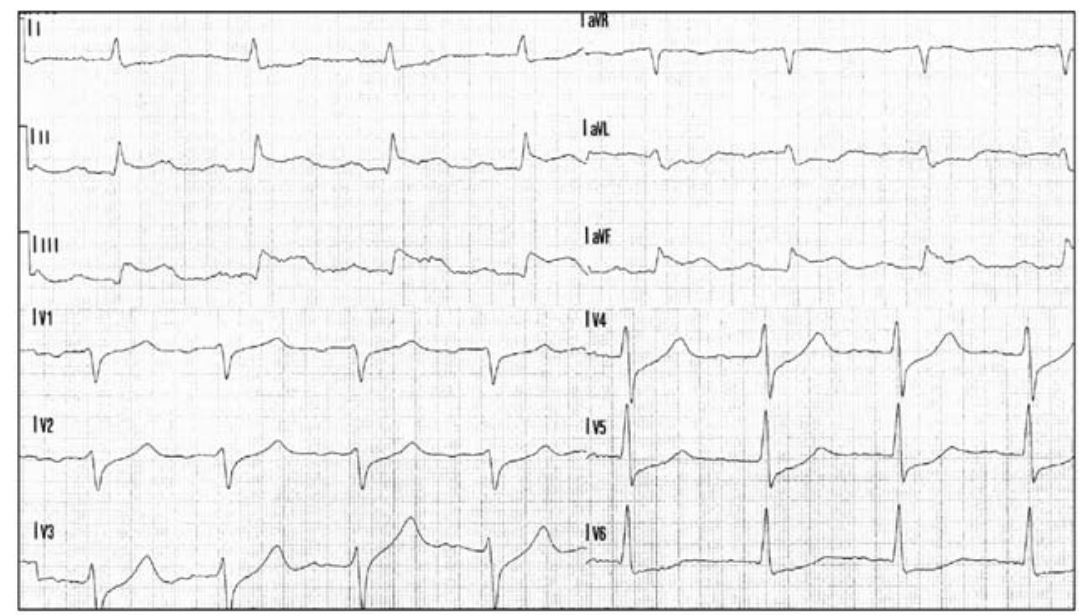

Figure 1. The ECG recorded in our emergency department indicated ST-elevation myocardial infarction
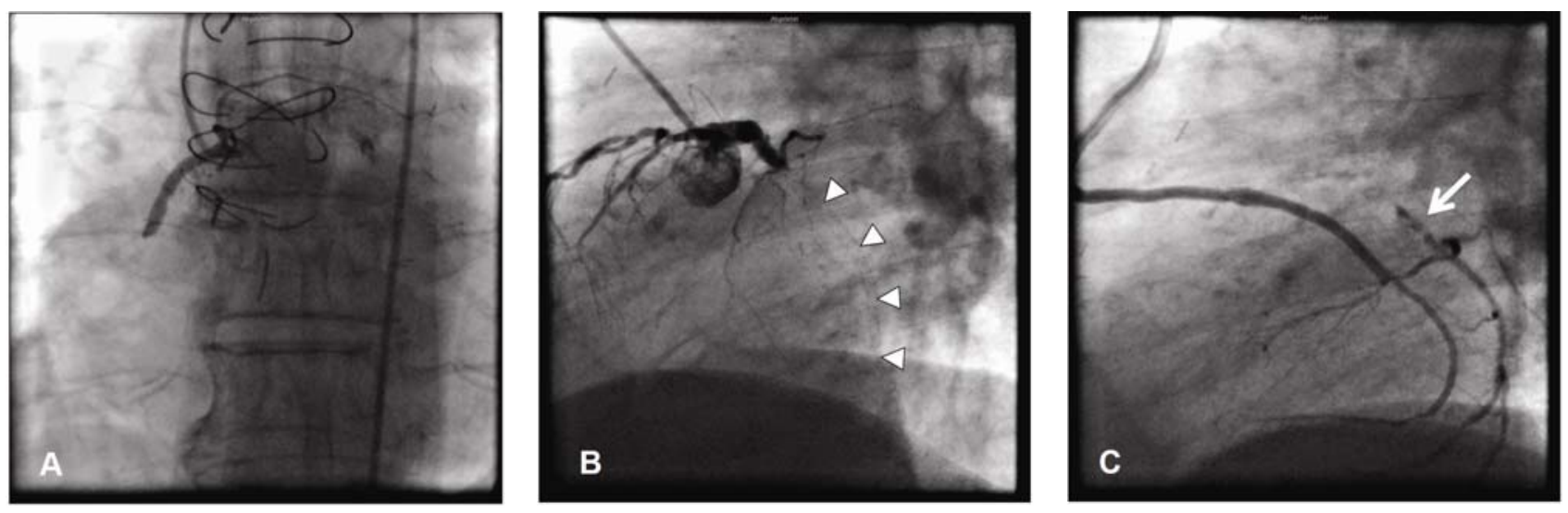

Figure 2. Angiography revealed an occlusion of the right coronary artery (A). In addition, a stent thrombosis of the left circumflex artery was apparent in the angiography of the left coronary artery (B) and of the saphenous bypass graft to the circumflex artery (C). 

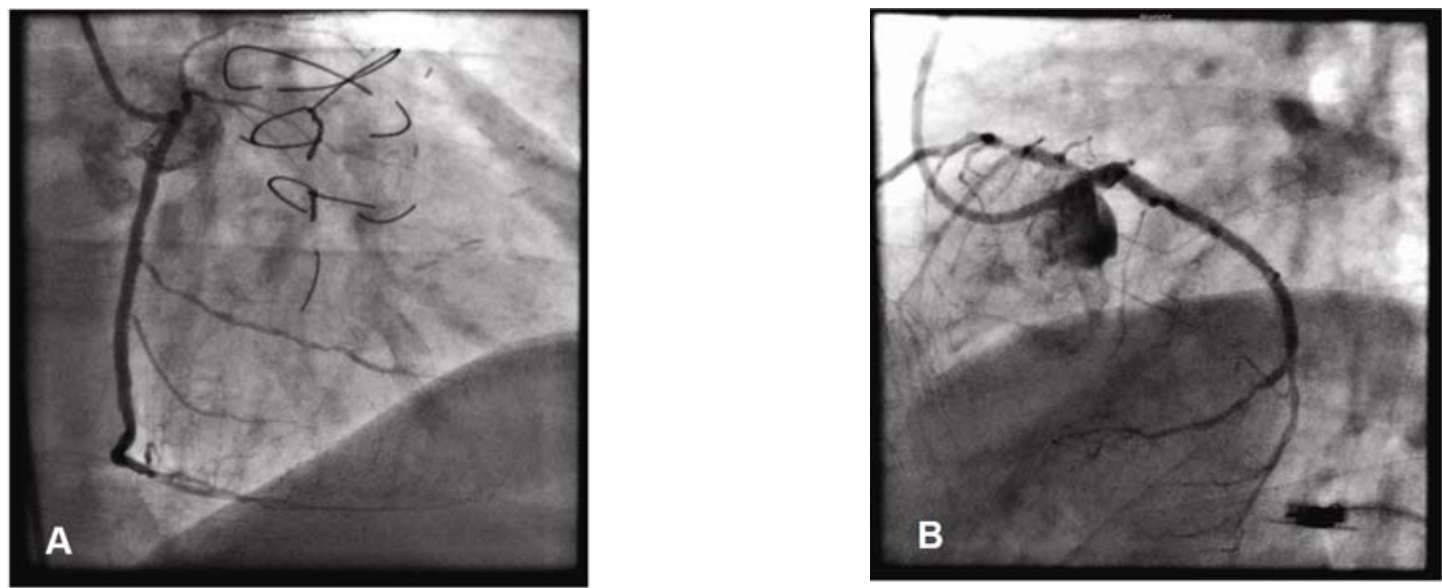

Figure 3. Results after stent implantation in the right coronary artery (A) and balloon angioplasty of the left circumflex artery (B)

\section{Discussion}

We speculate that the culprit lesion responsible for myocardial infarction was a plaque rupture in the RCA. Subsequently, altered hemodynamics and hypercoagulability presumably caused stent thrombosis in the LCx. Cardiogenic shock was prevented by the retrograde flow from the saphenous graft which was, at rest, sufficient to supply blood to the LCx-dependent myocardium - despite severe stenosis of the proximal segment of the first marginal branch.

In conclusion, STEMI patients are in a hypercoagulant state ${ }^{[1-3]}$ and therefore prone to additional thrombotic complications such as late stent thrombosis which requires adequate awareness of the interventionist. Recently published results from the PRAMI and CvLPRIT trials ${ }^{[4,5]}$ suggest that STEMI patients benefit from early complete revascularization compared to treatment of the culprit lesion only. This underscores the importance of a careful evaluation of all additional lesions. Our case report shows that this must include consideration of a possible late stent thrombosis even many years after initial stent implantation.

\section{Conflict of interest statement}

The authors have no conflict of interests to declare.

\section{References}

[1] Merlini PA, Bauer KA, Oltrona L, et al. Persistent activation of coagulation mechanism in unstable angina and myocardial infarction. Circulation. 1994; 90: 61-68. http://dx.doi.org/10.1161/01.CIR.90.1.61

[2] Ault KA, Cannon CP, Mitchell J, et al. Platelet activation in patients after an acute coronary syndrome: results from the TIMI-12 trial. Thrombolysis in Myocardial Infarction. J Am Coll Cardiol. 1999; 33: 634-39. http://dx.doi.org/10.1016/S0735-1097(98)00635-4

[3] Undas A, Szuldrzynski K, Brummel-Ziedins KE, et al. Systemic blood coagulation activation in acute coronary syndromes. Blood. 2009; 113: 2070-78. http://dx.doi.org/10.1182/blood-2008-07-167411

[4] Wald DS, Morris JK, Wald NJ, et al. Randomized trial of preventive angioplasty in myocardial infarction. Engl J Med. 2013; 369(12): 1115-23. http://dx.doi.org/10.1056/NEJMoa1305520

[5] Kelly DJ, McCann GP, Blackman D, et al. Complete Versus culprit-Lesion only PRimary PCI Trial (CVLPRIT): a multicentre trial testing management strategies when multivessel disease is detected at the time of primary PCI: rationale and design.

EuroIntervention. 2013; 8(10): 1190-8. http://dx.doi.org/10.4244/EIJV8I10A183 